\title{
Estimator Design Using Four Relative Displacement Sensors for Semi-Active Suspension System
}

\author{
Arjon Turnip ${ }^{1}$, Iwan R. Setiawan ${ }^{1}$, Hanif Fakhrurroja ${ }^{1}$, Taufik Hidayat ${ }^{1}$, and Keum-Shik Hong ${ }^{2}$ \\ ${ }^{1}$ Technical Implementation Unit for Instrumentation Development, \\ Indonesian Institute of Sciences, Bandung, Indonesia \\ ${ }^{2}$ School of Mechanical Engineering, Pusan National University, Korea
}

\begin{abstract}
Estimator designs only using relative displacement information for semi-active suspension system are investigated. Modified sensitivity method controls semiactive suspension directly using relative velocity and MR damper model without calculating absolute velocity and parameter estimator. The comfort evaluation parameters about the driver are presented and the mathematical models of skyhook and modified skyhook control suspension are simulated in Simulink. The comparison results of suggests that modified skyhook control could significantly improve riding comfort and provide more comfortable working conditions for passenger, which can be widely applied on car in the future.
\end{abstract}

Index Terms - Semi-Active Suspension, Skyhook Control, Estimator, MR damper, Full-vehicle Model, Relative Displacement Sensor

\section{INTRODUCTION}

In recent years requirements for increased process performance, ride comfort and safety have generated a demand for high performance vibration suppression methods [1-17]. There already exists a wide variety of methods and approaches to vibration mitigation, varying from the adaptive and nonlinear approaches to the linear feedback control via filtering and optimal state-feedback methods. This is associated with new solutions for innovative suspensions applied in vehicles, transport platforms, agricultural tractors, operator cabins and seats, etc. A suspension system performs two main functions. The first is to give support to the structure at the accepted static deflection. In addition, suspension systems in a car have to ensure good contact between the wheels and the surface of the road [4-6]. Thus the conventional suspensions are usually very stiff. The second function is associated with the vibroisolation of car bodies, operator seats, and platforms used to transport equipment sensitive to vibration. In this case, suspension should reduce vibration due to the external disturbances to the maximum possible degree. It is difficult to meet both requirements, since this involves opposing tendencies in the selection of the main parameters of suspension, i.e. stiffness and damping. Suspension should be rigid enough to effectively carry the static load, and soft enough to ensure the good isolation of vibrations. The design of suspension is always a compromise between safety and comfort. Several studies have shown that this conflict can be eased by using controlled suspension systems instead of passive ones. Especially semi-active dampers are wide spread in industrial application because of their performance increase compared to passive suspensions and their lower energy consumption compared to active systems [1]. Methods for controlling semi-active suspension systems reach back to the works of Karnopp who has proposed the skyhook algorithm in order to improve the ride comfort [3-5]. Several extensions of skyhook control have been suggested in the past decades and especially the combination of a skyhook-based controller with a groundhook algorithm promises additional improvements on ride safety [7-8].

The skyhook control is simple and provides a relatively good vibration suppression capability compared to other algorithms. The implementation of the skyhook control requires information on the relative velocity of the sprung and unsprung masses as well as the absolute velocity of the sprung mass [8]. To achieve this, at least seven acceleration sensors, if a full vehicle model is used, are needed. Because only four relative displacement sensors are used in this paper, three ways estimation schemes for the absolute velocity of the sprung mass is pursued. The skyhook control itself is good at suppressing the vibration of the sprung mass, however it deteriorates the vibration of the unsprung mass [9]. From this point of view, a weighted skyhook control for the purpose of improving the vibration suppression capability of the unsprung mass near $10 \mathrm{~Hz}$ is investigated. The contributions of this paper are the following. A new control strategy using four relative displacement sensors is pursued. The conventional skyhook control is modified in such a way that the control characteristics at $10 \mathrm{~Hz}$ is improved.

This paper is structured as follows. In Section 2, a fullvehicle model for the semi-active suspension system using MR damper is introduced. In Section 3, three ways estimator and weighted skyhook control algorithms are introduced. In Section 4, the performances of three ways estimator and a controlled semi-active suspension system 
are evaluated with Simulink. Conclusions are given in Section 5.

\section{MODELING OF A FULL-VEHICLE MODEL}

When the ride quality in various roads need to be improved, not sacrificing the handling performance of the vehicle with semi-active dampers, the damping force range needs to be widened and the response time of the dampers needs to become small. Hence, the use of a continuous damping control (CDC) damper is needed. A discrete damping control damper equipped with a step motor (or even a hydraulic damper equipped with a solenoid valve) is capable of controlling 1-4 Hz road disturbances [17]. Hence, on a road with high frequency components, the vibration suppression capability of the traditional hydraulic CDC damper is limited. One of the notable features in this paper is the use of relative displacement sensors, in contrast with the conventional method which uses two acceleration sensors on the sprung and unsprung masses, respectively. The measurement principle of relative displacement is to detect the phase difference between the input and output sinusoidal signals, which changes with the movement of the piston. With this approach, a relative displacement up to the size of micro-meters can be measured. Most of all, the sensing mechanism is resistant to external impacts and noises.

In this paper, however, because the absolute velocities at four corner points of the sprung mass (vehicle body) have to be estimated, a full-vehicle model is considered. If the vertical velocity at the center of the vehicle body ( $\left.\dot{v}_{s}\right)$ and the roll and pitch rates $(\dot{\phi}, \dot{\theta})$ are known, the velocities at the four corners points can be easily calculated using the geometry of the vehicle body. The accuracy of models can be enhanced by extending from a quarter-vehicle model to a full-vehicle model as given below. The improvement comes from the fact that the roll and pitch motions of the vehicle can be incorporated and the mass moment of inertia of the vehicle can be included [17].

$$
\begin{aligned}
m_{s} \ddot{z}_{s}= & -k_{s f}\left(z_{s f l}-z_{u f l}\right)-k_{s f}\left(z_{s f r}-z_{u f r}\right) \\
& -k_{s r}\left(z_{s r l}-z_{u r l}\right)-k_{s r}\left(z_{s r r}-z_{u r r}\right) \\
& -f_{f l}-f_{f r}-f_{r l}-f_{r r}, \\
I_{\theta} \ddot{\theta}= & k_{s f} l_{f}\left(z_{s f l}-z_{u f l}\right)+k_{s f} l_{f}\left(z_{s f r}-z_{u f r}\right) \\
& -k_{s r} l_{r}\left(z_{s r l}-z_{u r l}\right)-k_{s r} l_{r}\left(z_{s r r}-z_{u r r}\right) \\
& +l_{f} f_{f l}+l_{f} f_{f r}-l_{r} f_{r l}-l_{r} f_{r r}, \\
I_{\phi} \ddot{\phi}= & -k_{s f} t_{f}\left(z_{s f l}-z_{u f l}\right)+k_{s f} t_{f}\left(z_{s f r}-z_{u f r}\right) \\
& -k_{s r} t_{r}\left(z_{s r l}-z_{u r l}\right)+k_{s r} t_{r}\left(z_{s r r}-z_{u r r}\right) \\
& -t_{f} f_{f l}+t_{f} f_{f r}-t_{r} f_{r l}+t_{r} f_{r r} .
\end{aligned}
$$

The subscript appearing in (1): $s$ and $u s$ indicate sprung and unsprung mass; $f l, f r, r l$, and $r r$ are front left, front right, rear left, and rear right, respectively. A full-vehicle model depicts the schematic of a full-vehicle model and the control strategy using the relative displacement of the sprung and unsprung masses. Let $f$ be the force generated at the damper, which is the control force. Then the equations of motion are

$$
\begin{aligned}
& m_{u f} \ddot{z}_{u f l}=k_{s f}\left(z_{s f l}-z_{u f l}\right)-k_{t f}\left(z_{u f l}-w_{f l}\right)+f_{f l}, \\
& m_{u f} \ddot{z}_{u f r}=k_{s f}\left(z_{s f r}-z_{u f r}\right)-k_{t f}\left(z_{u f r}-w_{f r}\right)+f_{f r}, \\
& m_{u r} \ddot{z}_{u r l}=k_{s r}\left(z_{s r l}-z_{u r l}\right)-k_{t r}\left(z_{u r l}-w_{r l}\right)+f_{r l}, \\
& m_{u r} \ddot{z}_{u r r}=k_{s r}\left(z_{s r r}-z_{u r r}\right)-k_{t r}\left(z_{u r r}-w_{r r}\right)+f_{r r} . \\
& z_{s f l}=z_{s}+l_{f} \theta+t_{f} \phi, \\
& z_{s f r}=z_{s}+l_{f} \theta-t_{f} \phi, \\
& z_{s r l}=z_{s}-l_{r} \theta+t_{r} \phi, \\
& z_{s r r}=z_{s}-l_{r} \theta-t_{r} \phi .
\end{aligned}
$$

\section{MODIFIED SKYHOOK CONTROL}

In this section, upon the use of relative displacement sensors, the skyhook control approach introduced by Karnopp et al. (1974) for a quarter model is applied to a full-vehicle model by a modification of the law to improve the handling performance. The skyhook approach means inserting a fictitious damper between the sprung mass and an imaginary sky so that the vibration of the sprung mass is damped out through the imaginary damper. The skyhook control is used to reduce the vertical acceleration of the sprung mass for the ride quality improvement. The skyhook control law is concise and is known adequate for a 8-bit ECU due to its computational simplicity, while the performance is almost equivalent to a state feedback control. However, the implementation of a skyhook control requires the velocity of the sprung mass as well as the relative velocity between the sprung and unsprung masses. For completeness of the paper, the skyhook control law is reviewed first as follows.

$$
\begin{aligned}
& f_{j}=-c_{s, j} \cdot\left(\dot{z}_{s, j}-\dot{z}_{u, j}\right) \quad, \quad \text { if } \quad \dot{z}_{s, j}\left(\dot{z}_{s, j}-\dot{z}_{u, j}\right)>0, \\
& f_{j}=\min _{I}\left(c_{s, j}(I)\right) \cdot\left(\dot{z}_{s, j}-\dot{z}_{u, j}\right), \text { if } \dot{z}_{s, j}\left(\dot{z}_{s, j}-\dot{z}_{u, j}\right)<0,(4) \\
& \text { where } c_{s k y, j}=2 \varsigma_{\text {critical }} \sqrt{\left(m_{s} / 4\right) k_{s, j}}, j \in\{f l, f r, r l, r r\}
\end{aligned}
$$

(i.e., $j$ refers to the four dampers), $c_{s k y, j}$ is the desired skyhook damping coefficient at the $j$-th damper, $\varsigma_{\text {critical }}$ is the critical damping ratio, and $I$ is the current input to the damper.

The above skyhook control law is very effective in reducing the resonance peak of the sprung mass, but it deteriorates the handling performance by reducing the contacting force between the tire and the road surface at the resonance frequency of the unsprung mass. In order to overcome this, the skyhook control is modified as follows: We first filter the signals via a band-pass filter of 8-12 Hz, which is the resonance frequency band of the unsprung mass, and changes the skyhook damping coefficient $c_{s k y, j}$ in relation to the magnitude of the 
filtered signal, and then the damping force of the MR dampers is increased when the road disturbance is given in the range of the unsprung mass resonance frequency. The relative velocities between the sprung mass and four unsprung masses are obtained by differentiating the relative displacement signals that were processed with a band pass filter as follows.

$$
\dot{z}_{s, j}-\dot{z}_{u, j}=s \cdot \frac{40 \omega_{b} s}{s^{2}+40 \omega_{b}+\omega_{b}^{2}}\left(z_{s, j}-z_{u, j}\right),
$$

where $j \in\{f l, f r, r l, r r\}$ and $\omega_{b}$ is a design parameter which characterizes the band $\omega_{b}=10 \mathrm{~Hz}$ ).

$$
c_{\text {mod }, j}=2 \varsigma_{\text {critical }} \sqrt{\left(m_{s} / 4\right) k_{s, j}}+\beta \cdot\left(\dot{z}_{s, j}-\dot{z}_{u, j}\right)
$$

where $\beta$ is a controller parameter, which needs to be tuned for a given vehicle (in our case, $\beta=1.2$ is used). The idea in this modification is to make the MR damper harder by adding the second term in (6) that focuses on the resonance frequency of the unsprung mass. Because $\left(\dot{z}_{s, j}-\dot{z}_{u, j}\right)$ is obtained through the band pass filter in (5), the road disturbance at $10 \mathrm{~Hz}$ affects the second term in (6). The role of $\beta$ is to provide a freedom to the driver to choose the ride quality or the handling performance of the given suspension system. Finally, the following modified skyhook law is proposed.

$$
\begin{aligned}
& f_{j}=-c_{\bmod , j} \cdot \dot{z}_{s, j} \quad, \quad \text { if } \quad \dot{z}_{s, j}\left(\dot{z}_{s, j}-\dot{z}_{u, j}\right)>0 \\
& f_{j}=\min _{I}\left(c_{s, j}(I)\right) \cdot \dot{z}_{s, j}, \text { if } \dot{z}_{s, j}\left(\dot{z}_{s, j}-\dot{z}_{u, j}\right)<0 .
\end{aligned}
$$

Since $\dot{z}_{s, j}$ in (8) can not be measured, two estimation schemes for it are discussed in the next section.

\section{Full-Vehicle Model Estimator}

The first approach is to use the equations of motion of the full-vehicle model developed in the previous section. $\dot{z}_{s, j}$ can be calculated using the geometry of the vehicle body (i.e., $t_{f}, t_{r}, l_{f}, l_{r}$ ), if $\dot{z}_{s}, \dot{\phi}$, and $\dot{\theta}$ are known. Therefore, motivated by (1), the use of the following equations is proposed.

$$
\begin{aligned}
\ddot{\hat{z}}_{s} & \cong \frac{1}{m_{s}}\left[-k_{s f}\left(z_{s f l}-z_{u f l}\right)-k_{s f}\left(z_{s f r}-z_{u f r}\right)\right. \\
& -k_{s r}\left(z_{s r l}-z_{u r l}\right)-k_{s r}\left(z_{s r r}-z_{u r r}\right) \\
& \left.-f_{f l}-f_{f r}-f_{r l}-f_{r r}\right] \\
\ddot{\hat{\theta}} \cong & \frac{1}{I_{\theta}}\left[k_{s f} l_{f}\left(z_{s f l}-z_{u f l}\right)+k_{s f} l_{f}\left(z_{s f r}-z_{u f r}\right)\right. \\
& -k_{s r} l_{r}\left(z_{s r l}-z_{u r l}\right)-k_{s r} l_{r}\left(z_{s r r}-z_{u r r}\right) \\
& \left.+l_{f} f_{f l}+l_{f} f_{f r}-l_{r} f_{r l}-l_{r} f_{r r}\right]
\end{aligned}
$$

$$
\begin{aligned}
\ddot{\hat{\phi}} \cong & \frac{1}{I_{\phi}}\left[-k_{s f} t_{f}\left(z_{s f l}-z_{u f l}\right)+k_{s f} t_{f}\left(z_{s f r}-z_{u f r}\right)\right. \\
& -k_{s r} t_{r}\left(z_{s r l}-z_{u r l}\right)+k_{s r} t_{r}\left(z_{s r r}-z_{u r r}\right) \\
& \left.-t_{f} f_{f l}+t_{f} f_{f r}-t_{r} f_{r l}+t_{r} f_{r r}\right]
\end{aligned}
$$

where $\ddot{\hat{z}}, \ddot{\hat{\theta}}, \ddot{\hat{\phi}}$ denote the estimates of $\ddot{\hat{z}}, \ddot{\hat{\theta}}, \ddot{\hat{\phi}}$ respectively. In (8), because the real damping force $f_{j}$ of the MR dampers can not be measured, the command signal $f_{j}$ in (7) is replaced under the assumption that the command signal $f_{j}$ is well generated through the real dampers.

With (8), the estimates of the velocities $\left(\dot{\hat{z}}_{s}, \dot{\hat{\phi}}\right.$, and $\left.\dot{\hat{\theta}}\right)$ are now obtained by integrating (8) and passing through a high pass filter as follows.

$$
\begin{aligned}
& \dot{\hat{z}}_{s}=\frac{s}{s+1.256} \cdot \frac{1}{s} \cdot \ddot{\hat{z}}_{s}, \\
& \dot{\hat{\theta}}=\frac{s}{s+1.256} \cdot \frac{1}{s} \cdot \ddot{\hat{\theta}}, \\
& \dot{\hat{\phi}}=\frac{s}{s+1.256} \cdot \frac{1}{s} \cdot \ddot{\hat{\phi}},
\end{aligned}
$$

where a high pass filter of $0.2 \mathrm{~Hz}$ cutoff frequency is used. This high pass filter removes the DC offset component (integration constant). Fig. 1 shows the comparison of the unsprung mass displacements. Finally, using (3), the absolute velocities of the four corner poipts are obtained as follows. Fig. 2 shows the comparison of the sprung mass velocities. The Simulink simulation diagram is given in Fig. 3.

$$
\begin{aligned}
& \dot{\hat{z}}_{s, f l}=\dot{\hat{z}}_{s}+l_{f} \dot{\hat{\theta}}+t_{f} \dot{\hat{\phi}}, \\
& \dot{\hat{z}}_{s, f r}=\dot{\hat{z}}_{s}+l_{f} \dot{\hat{\theta}}-t_{f} \dot{\hat{\phi}}, \\
& \dot{\hat{z}}_{s, r l}=\dot{\hat{z}}_{s}-l_{f} \dot{\hat{\theta}}+t_{f} \dot{\hat{\phi}}, \\
& \dot{\hat{z}}_{s, r r}=\dot{\hat{z}}_{s}-l_{f} \dot{\hat{\theta}}-t_{f} \dot{\hat{\phi}} .
\end{aligned}
$$

The effects of the road excitation frequency and road roughness in association with the ride comfort and the road holding of the vehicle were studied. To find the best compromise for the trade-off between the ride comfort and road holding effects, a modified skyhook control was developed (i.e., the two independent variables to be specified by designers can be adaptively tuned to precisely achieve the type of desired response). The magnitudes of the frequency responses of the sprungmass acceleration with respect to the road disturbance are shown in Fig. 4. The red line and the blue line represent the magnitude plots of the frequency responses of the modified and conventional skyhook methods, respectively. In Fig. 4, the red line depicts an improvement in the frequency range 4-8 $\mathrm{Hz}$. The simulation result show that a semi-active suspension system with the proposed control strategy is able to 
improve the ride comfort as shown in Fig. 4 and the road holding as shown in Fig. 5, significantly compared with the conventional skyhook control. Around $6 \mathrm{~Hz}$ frequency ranges, the performance of conventional skyhook is slightly better than the midified one. It is predicted as one limitation of the estimator design.

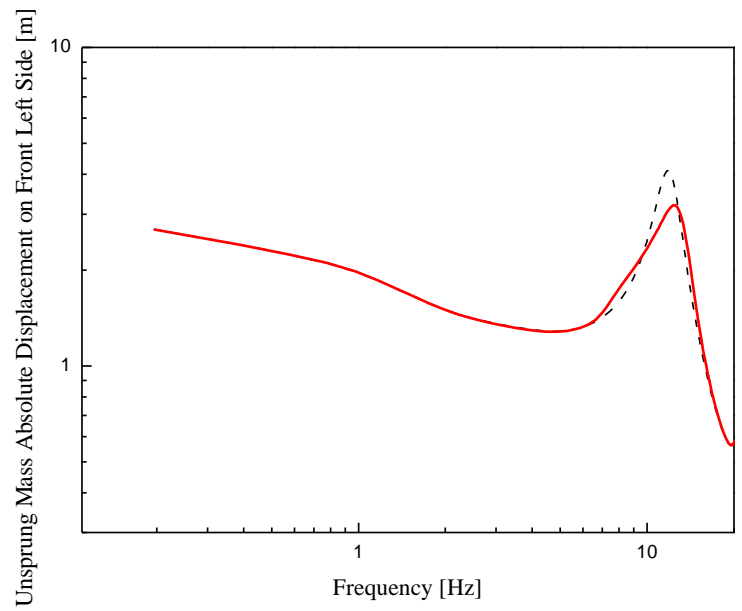

Fig. 1 Comparison of the unsprung mass displacements: Skyhook control (dashed line)vs. modified skyhook control (solid line)

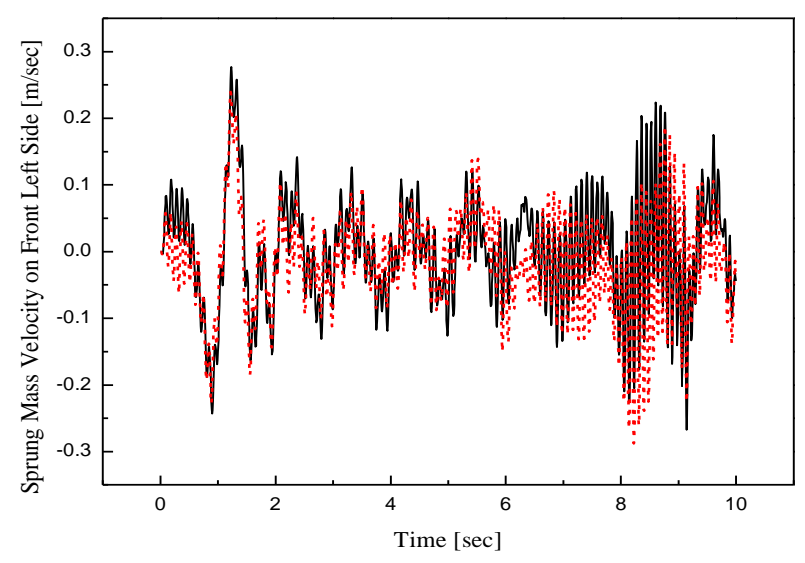

Fig. 2 Comparison of the sprung mass velocities: The estimated velocity using the equations of motion (dot line) vs. the real one (solid line)

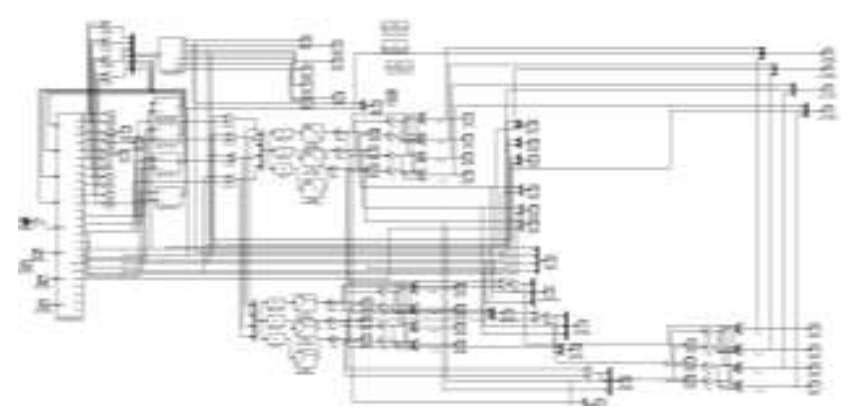

Fig. 3 Simulink Diagram for Simulation

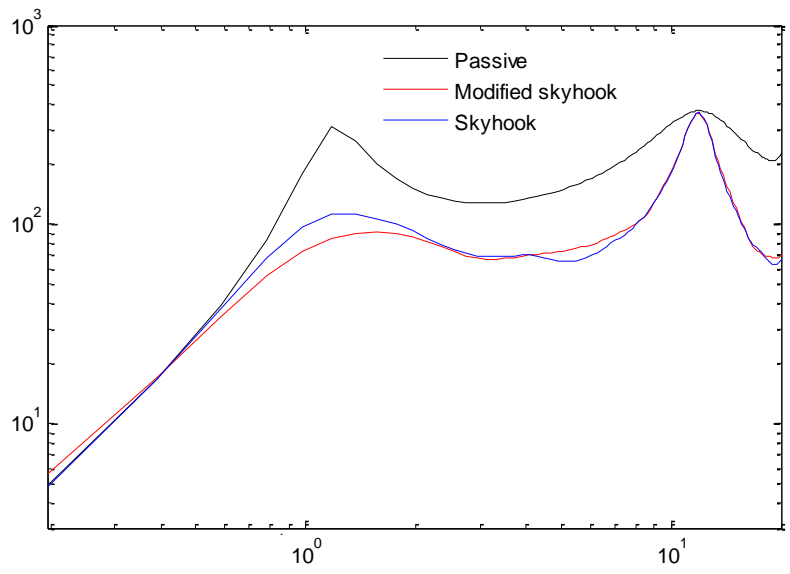

Fig. 4 Frequency response of sprung mass absolute acceleration (front left side): Passive (black line), skyhook control (blue line), and modified skyhook control (red line)

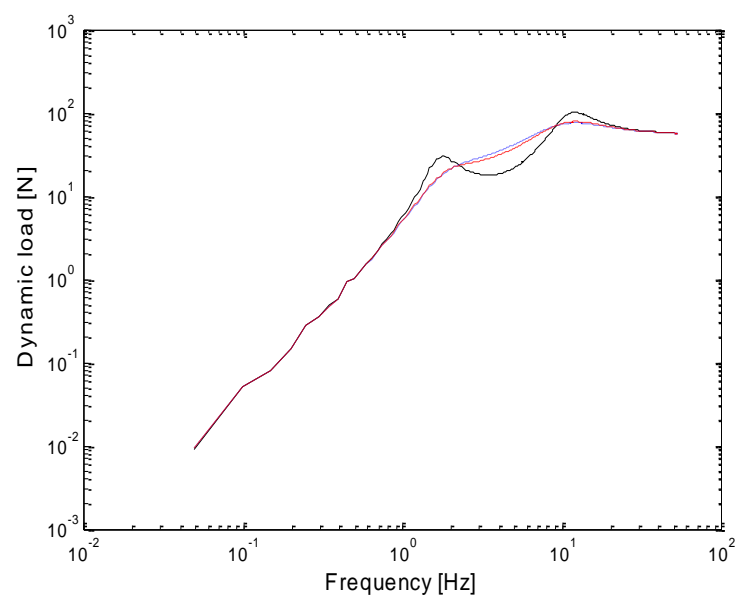

Fig. 5 Frequency responses of dynmic $\operatorname{load}(s) / z_{r}(s)$ (front left side): passive (black line), skyhook control (blue line), and modified skyhook control (red line)

\section{CONCLUSION}

For improving the handling performance of the vehicle near the resonance frequency of the unsprung mass, the traditional skyhook coefficient was modified resulting in the addition of a relative velocity term to the critical damping ratio. For estimating the absolute velocities of the four corner points of the sprung mass, a full-vehicle model that describes the heave, roll, and pitch motions of the vehicle body was utilized. The simulation result show that the sprung mass acceleration (i.e. indicate the ride comfort) and the dynamic load (i.e. indicate the road holding) especially at the resonance frequencies were significantly improve.

\section{ACKNOWLEDGMENT}

This research was supported by the thematic program through the Bandung Technical Management Unit for Instrumentation Development (Deputy for Scientific Services) and the flagship program through the Research Center for Physics (Deputy for Engineering Sciences) funded by Indonesian Institute of Sciences, Indonesia. 


\section{REFERENCES}

[1] E. Guglielmino, T. Sireteanu, C. W. Stammers, G. Ghita and M. Giuclea: Semiactive suspension control, (2008), Springer Verlag London Limited.

[2] A. Turnip, K.-S. Hong and S. H. Park: Modeling of Hydraulic Engine Mount for Active Pneumatic Engine Vibration Control Using the Extended Kalman Filter, Journal of Mechanical Science and Technology, vol.23, no.1, (2009) p.229-236.

[3] D. Karnopp: Active damping in road vehicle suspensions, Vehicle System Dynamics, 12, (1983), p. 291-316.

[4] D. Karnopp, M. J. Crosby and R. A. Harwood: Vibration control using semi-active for generators, SME Journal of Engineering for Industry, 96 (May), (1974), p. 619-626.

[5] D. Karnopp and G. Heess: Electronically controllable vehicle suspensions, Vehicle System Dynamics, 20 (May), (1991), p. 207-217.

[6] A. Turnip and K.-S. Hong: Road-frequency based optimization of damping coefficients for semi-active suspension systems, International Journal of Vehicle Design, vol. 63, no. 1, (2013) p. 84-101.

[7] M. Ahmadian, X. Song and S. C. Southward: No-jerk skyhook control methods for semi-active suspensions, Journal of Vibration and Acoustics, 126(4), (2004), p. 580-584.

[8] A. Turnip, S. H. Park and K.-S. Hong: Sensitivity Control of a MR-Damper Semi-Active Suspension, International Journal of Precision Engineering and Manufacturing, vol.11, no.2, (2010) p.5-13.

[9] M. Ahmadian and A.P. Christopher: A Quarter-Car Experimental Analysis of Alternative Semi-Active Control Methods, Journal of Intelligent Material Systems and Structures, Vol. 11, (2000), p. 604-612.
[10] A. S. Cherry, R. P. Jones and T. E. C. Potter: The Use of Multibody System Modeling and Multivariable System Decoupling Technique in Vehicle Ride Control," ASME Transactions, Journal of Dynamic Systems, Measurement, and Control, Vol. 121, No. 3 (1999), p. 479-486.

[11] A. Turnip, D. Soetraprawata, Hariyadi and D.E. Kusumandari: Design of an adaptive intelligent controller in a semi-active suspension systems, Teknologi Indonesia, vol. 36, no. 1 (2013), p. 9-15.

[12] S. B. Choi, S. K. Lee and Y. P. Park: A Hysteresis Model for the Field-Dependent Damping Force of a Magnetorheological Damper, Journal of Sound and Vibration, Vol. 2, (2011), p. 375383.

[13] F. D. Goncalves and M. Ahmadian: A Hybrid Conrol Policy for Semi-Active Vehicle Suspensions, Shock and Vibration, Vol. 10, (2003), p. 59-69.

[14] A. Turnip and H. Fakhrurroja: Estimation of the wheel-ground contact tire forces using extended Kalman Filter, International Journal of Instrumentation Science, vol. 2, no. 2 (2013), p. 34.

[15] K. S. Hong, D. S. Jeon, W. S.Yoo, H. Sunwoo, S. Y. Shin, C. M. Kim and B. S. Park: A New Model and an Optimal Poleplacement Control of the Macpherson Suspension System, SAE International Congress and Exposition, Detroit, MI, SAE paper No. 1999-01-1331, (1999), p. 267-276.

[16] Y. Liu, T. P. Waters and M. J. Brennan: A Comparison of SemiActive Damping Control Strategies for Vibration Isolation of Harmonic Disturbances, Journal of Sound and Vibration, Vol. 280, (2005), p. 21-39.

[17] D.-M. Oh, A. Turnip, K.-S. Hong: Control Strategies of a Semi-Active Suspension System Using Four Relative Displacement Sensors, International Symposium on Advanced Vehicle Engineering, 2008. 